\title{
A Self-Powered Medical Device for Blood Irradiation Therapy
}

\author{
Avigail D. Amsel, Arkady Rudnitsky, and Zeev Zalevsky \\ Faculty of Engineering, Bar Ilan University, Ramat Gan 52900, Israel \\ Correspondence should be addressed to Avigail D. Amsel, a.avigail@gmail.com and Zeev Zalevsky, zalevsz@biu.ac.il
}

Received 4 March 2012; Accepted 7 May 2012

Academic Editor: Pietro Ferraro

Copyright ( 2012 Avigail D. Amsel et al. This is an open access article distributed under the Creative Commons Attribution License, which permits unrestricted use, distribution, and reproduction in any medium, provided the original work is properly cited.

\begin{abstract}
Implantable wireless devices may allow localized real-time biomedical treating and monitoring. However, such devices require a power source, which ideally, should be self-powered and not battery dependent. In this paper, we present a novel self-powered light therapeutic device which is designed to implement blood irradiation therapy. This device is self-powered by a miniaturized turbine-based generator which uses hydraulic flow energy as its power source. The research presented in this paper may become the first step towards a new type of biomedical self-operational micromechanical devices deployed for biomedical applications.
\end{abstract}

\section{Introduction}

Implantable wireless devices may allow localized real-time biomedical treating and monitoring. However, such devices require a power source, which ideally, should be self-powered and not battery dependent. A human body holds within it a broad variety of potential power sources. This includes mechanical energy (in the form of body movements, muscle stretching, and blood vessel contractions) and hydraulic energy (in the form of blood flow). However, it is of great challenge to efficiently convert these power sources into electrical energy.

Over the last years, great progress has been made in the field of endovascular intervention as various intravascular implantable devices and techniques have been developed. This includes numerous devices used for mechanical vascular repair as well as new technologies for better treatment and diagnostics.

Balloon angioplasty, a technique used for mechanically widening a narrowed blood vessel, represents the beginning of a new era of treating cardiovascular disease. However, restenosis (renarrowing of vessels) and arterial lesions that are likely to dissect after angioplasty led to the development and usage of stents [1]. To date, it is still unknown whether stenting improves long-term clinical outcomes as compared with standard balloon angioplasty, as restenosis and other related complications continue to occur after both procedures [2]. Hence, the ongoing search for a proper solution has not yet come to an end and major effort has been and is still invested in order to improve stent design and engineering [3]. These efforts led to an evolution in regard to stent development starting from classical bare metal stents through the introduction of drug eluting stents, which are stents designed to reduce restenosis by eluting active substances over time [4]. Recently, a new generation of stents has been developed, for example, absorbable stents which mechanically support the vessel during the period of high risk for recoil and then completely degrade in the longterm perspective thereby avoiding the potential long-term complications of metal stents [5].

A great deal of progress has also been made in regard to intravascular implantable devices which are not directly associated with mechanical vascular repair. These systems are designated to allow better treatment and diagnosis of various clinical problems. A good example for such a development is the implantable pressure sensor "EndoSure" that was recently introduced by CardioMEMS, Inc., Atlanta, GA, which allows real-time measurements of intrasac pressure after endovascular aneurysm repair (EVAR). This device not only provides enhanced sensitivity to identify problems associated with EVAR failure, but also allows continuous surveillance and is potentially more cost-effective than other techniques which primarily consist of CT (computerized tomography) scanning with intravenous contrast $[6,7]$. 
Light irradiation of the blood, a method also known as photochemotherapy, has a wide range of biomedical effects which include immunomodulation, vasodilation, and antiarrhythmic [8] and antihypoxic effects [9]. There are many versions of this method depending on the physical parameters of the light used for the irradiation (laser and nonlaser as well as light sources at different wavelengths) and by the way of irradiation, that is, intravenous or transcutaneous irradiation [10].

Intravenous laser blood irradiation (IVLBI) therapy was first introduced by Meshalkin in 1981 [11]. Originally, this method was developed for the treatment of cardiovascular diseases. Since then, comprehensive research on IVLBI has been done, revealing its effects on various systems including the hematologic and immunologic systems. In this regard, IVLBI has been shown to have a positive influence on rheological properties of the blood; for example, it leads to a decrease in blood viscosity [12], which is of great interest to surgery and cardiology. In addition, helium-neon ( $\mathrm{He}-\mathrm{Ne}$ ) laser irradiation induced an increase in phagocytic activity and structural modulation of macrophages $[13,14]$ as well as proliferation of lymphocytes $[15,16]$.

As previously mentioned, irradiation of the blood is commonly administered either intravenously or through a transcutaneous irradiation, while each method has its advantages as well as downsides. While transcutaneous irradiation can be performed repetitively, is more patient friendly, and does not include penetration to the tissue, its major disadvantages are that it includes irradiation of nearby tissue (skin, nerves, and mussels) and requires a higher irradiation power (due to massive absorption). On the other hand, IVLBI includes insertion of a small catheter to a vein, from which light would be administered. This kind of procedure requires a much lower irradiation power; however, it involves inconveniency to the patient, higher risk for infections, requires medical skills and overall canot be performed frequently. These limitations can be partially overcome by inserting a self-powered irradiation devise inside a blood vessel. This way, blood would be directly illuminated thus require low level of irradiation and since the device is self-powered, there would be no need to go through repetitive procedures in order to replace the device or to perform a longer or higher frequently therapy.

As previously explained, blood irradiation therapy can be applied not only by laser light but also with nonlaser light sources. In 1986, Karandashov and coworkers first applied blue light for irradiation of the blood in patients with hypertension and angina pectoris [17]. Over the years, numerous clinical studies were published in regard to the impact that blue light blood irradiation (BLBI) has on blood lipid composition. It was shown that BLBI induces a significant decrease in the levels of total cholesterol and lowdensity lipoproteins (LDL) and an increase in the content of high-density lipoproteins (HDL) [18]. These findings are with no doubt of great significance to the treatment of coronary heart disease and atherosclerosis.

In general, a typical intravenous blood irradiation therapy includes light administration to a vein via a catheter and irradiation of the blood with a relatively low-power light

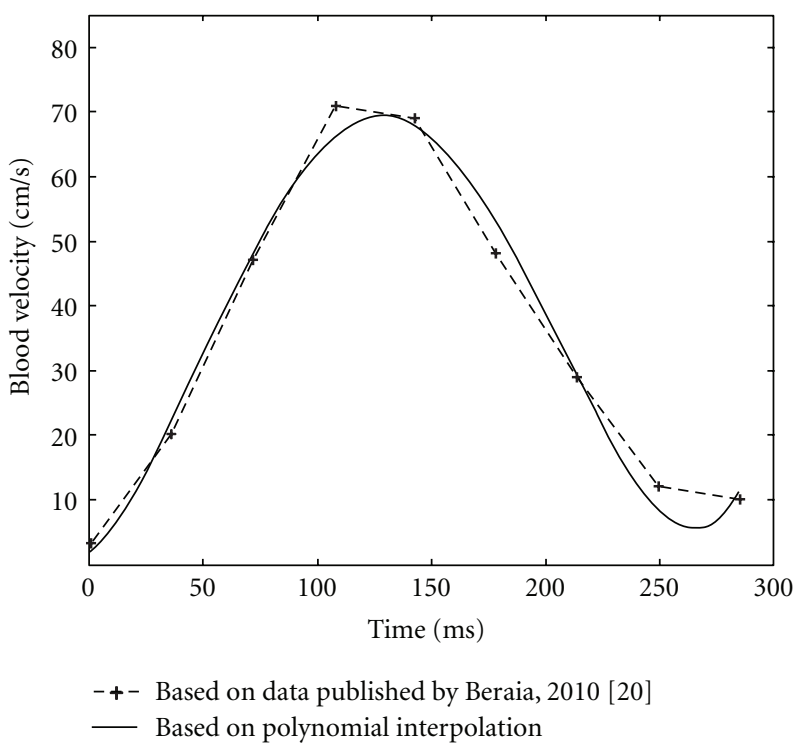

FIGURE 1: Blood velocity in ascending aorta during a heart beat. We show the comparison between polynomial function based on polynomial interpolation (solid line) and blood velocity graph based on data published in [20] (dashed line).

source for half an hour or so. Such a procedure should be carried out once or twice a day during a period of several days. An example commonly being used of such a therapy is blood irradiation with the He-Ne laser $(632.8 \mathrm{~nm})$ at a power of 1-3 mW, for 20-60 minutes once a day for up to ten days [19].

In this paper, we present a novel self-powered light therapeutic device which is designed to implement blood irradiation therapy. This device is self-powered by a miniaturized turbine-based generator which uses hydraulic energy in the form of blood flow as its power source.

\section{Design Computations}

It is a known fact that blood velocity depends on many factors including heart pulsation, type of blood vessel (artery or vein), vessels' width, and metabolic state (resting or exercising). However, during the following preliminary stages, we have performed several approximations related to a specific blood vessel as well as a steady metabolic state. Based on an article published by Beraia in 2010 [20], we have gathered data on different blood velocities at the ascending aorta during a heart beat. Using these blood velocities, we have built a graph which demonstrates blood velocity over the course of a heart beat. This graph is presented as the dashed line in Figure 1. One may see that this graph presents only a partial heart beat inasmuch as in normal conditions, a single heart beat takes more than $285 \mathrm{msec}$. However, during the rest of the beat, blood velocity was about zero. At this point, our main goal is to calculate the electric power that can be produced by blood flow. Since blood velocity during the rest of the beat is about zero, or in other words can not be 


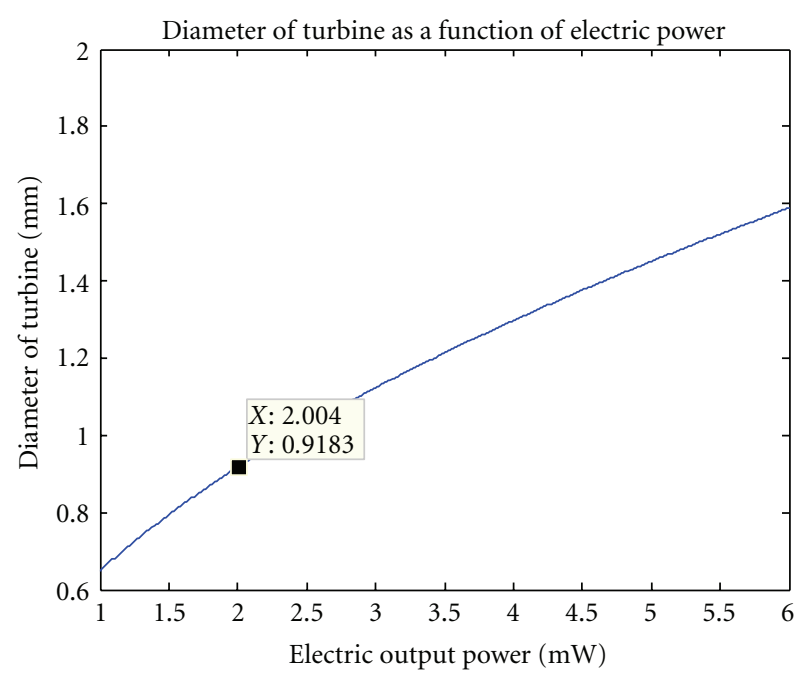

FIgUre 2: Turbines' diameter as a function of electric output power.

utilized to generate electric power, this part of the beat can be excluded from our calculations.

After describing blood velocity during a heart beat in a graphical manner, we have looked for a mathematical expression that matches this graph. In other words, we have searched for the best polynomial fit to the dashed curve of Figure 1. In order to do so, we have used MATLAB software and performed a polynomial interpolation. The result of this interpolation led to the following polynomial function:

$$
\begin{aligned}
v(t)= & 1.8107 \cdot 10^{-7} t^{4}-9.238 \cdot 10^{-5} t^{3}+0.0107 t^{2}+0.2894 t \\
& +1.6122 .
\end{aligned}
$$

In order to examine whether the function we found indeed matches the dashed graph of Figure 1, we have built a curve of $v(t)$ that is based on the polynomial approximation for temporal range of 1 to $285 \mathrm{msec}$. The polynomial fit curve is presented as the solid curve in Figure 1.

As expected, the level of compatibility between the polynomial function we found and the original dashed graph of velocity is relatively high with $R^{2}$ (coefficient of determination) of $98.5 \%$. Therefore, the function we found for describing blood velocity during a heart beat is quite reliable and can indeed be used in our future calculations.

In noncompressible fluid dynamics, dynamic pressure (indicated with $P_{d}$ ) is defined by [21]

$$
P_{d}=\frac{1}{2} \rho v^{2}(t),
$$

where $\rho$ is the fluid density (in $\mathrm{kg} / \mathrm{m}^{3}$ ) and $v$ is the fluid velocity (in $\mathrm{m} / \mathrm{s}$ ). Dynamic pressure can be used in order to define force (indicated with $F(t)$ ) that is applied on a turbine by a flow of a liquid, as given in the following equation:

$$
F(t)=S \cdot P_{d}=S \cdot \frac{\rho v^{2}(t)}{2},
$$

where $S$ represents a cross-section area $\left(\right.$ in $\mathrm{m}^{2}$ ).
In regard to (3), it is important to explain why we have assumed that blood velocity over the turbines' cross-section is uniform (despite the fact that usually, in tubes, the velocity distribution is nonuniform having low values at the edges and high at the center). The reason for doing so relates to the turbine's diameter, which is designed to be very small. In this case, it is relatively fair to approximate the blood velocity over such a small cross section as uniform.

We denote by $d l$ the distance which blood travels during a time unit (marked by $d t$ ):

$$
d l=v(t) \cdot d t .
$$

Mechanical work during a time unit $(d t)$ is defined by:

$$
d W=F(t) \cdot d l=F(t) \cdot v(t) \cdot d t .
$$

Therefore, the total work made by blood flow during a single heart beat is indicated with $W$ and equals

$$
W=\int_{1}^{285} F(t) \cdot v(t) \cdot d t=\frac{S \rho}{2} \int_{1}^{285} v^{3}(t) \cdot d t
$$

where the boundaries of the integration are in units of msec.

Finally, electric power (indicated with $P$ ) is defined by

$$
P(T)=\frac{d W}{d T},
$$

where $T$ stands for the time during which the work was done, and in our case it equals to the duration of a single heart beat, that is, the time between two sequential heart beats (as opposed to $t$, which represents the time during which work was calculated).

At this point, it is important to explain that the device we aim to develop is designed to operate in such a manner that first, electric power will be accumulated and then, it will consume this power for different applications (e.g., illumination). When referring to the way of which electrical power will be used, it can be described as a linear function. This is why (7) can be written as follows:

$$
P=\frac{W}{T} .
$$

By combining all these equations, one can represent the electric power by the following equation:

$$
P=\frac{S \rho}{2 T} \cdot \int_{1}^{285} v^{3}(t) \cdot d t
$$

or when presenting the area as a free parameter:

$$
S=\frac{2 P T}{\rho \cdot \int_{1}^{285} v^{3}(t) \cdot d t} .
$$

Assuming that the turbine area has circular cross section, turbines' diameter (indicated with $D$ and in units of $\mathrm{mm}$ ) will be calculated as follows:

$$
D=2 \cdot \sqrt{\frac{S}{\pi}} \cdot 10^{3} .
$$




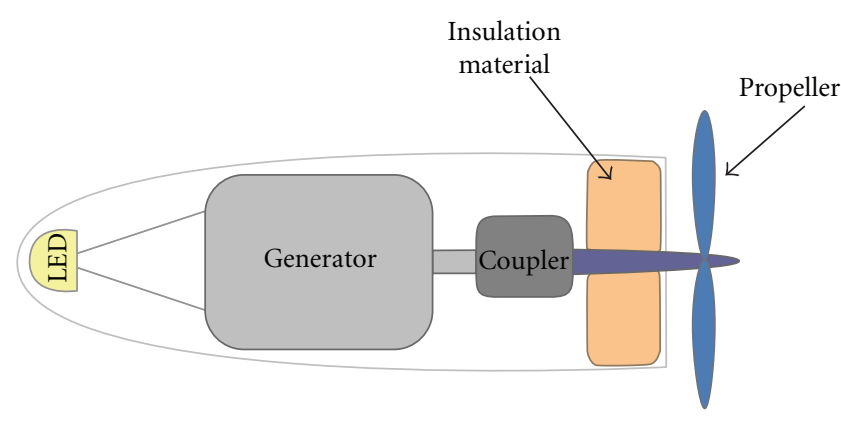

(a)

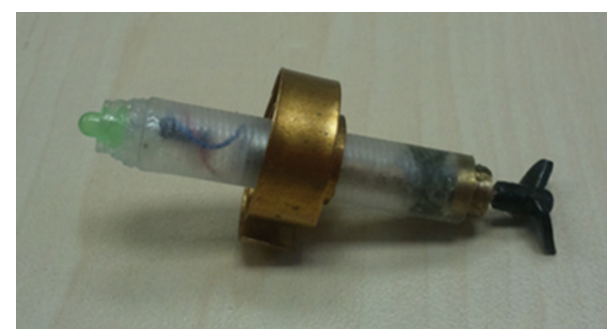

(b)

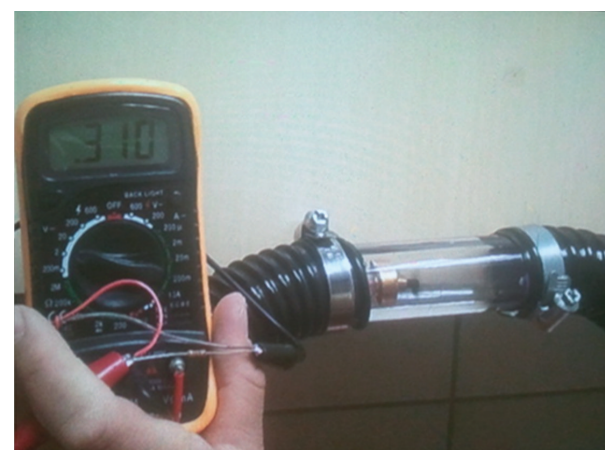

(c)

FIGURE 3: Intravascular implantable light therapeutic prototype. (a) Initial scheme of intravascular implantable light therapeutic system. (b) An image of the prototype. (c) Device inside a Perspex tube and a voltage of $0.3 \mathrm{~V}$ that was measured during water flow.

\section{Preliminary Experimental Investigation}

An initial step in our research included the use of (10) and (11) along with real parameters regarding blood flow in order to investigate feasibility. At this point, we have tried to examine whether blood velocity and density are sufficient to generate enough electrical power to operate a LED light source and to see whether this can be achieved by using a turbine with a diameter small enough to be located inside a blood vessel.

In regard to the electrical power which will be used during the following calculation, it is important to take into account the power efficiency of LED sources. There are many types of LED sources which consume different electrical power. At low currents, there are certain LEDs which exhibit power efficiency of about $12 \%$ [22]. This means that in order to illuminate at a power of $1 \mathrm{~mW}$, we need to supply electric power of about $8.3 \mathrm{~mW}$. Thus, if one is interested in illuminating at a power ranging between $1 \mathrm{~mW}$ and $6 \mathrm{~mW}$, the required electrical power will range from about $8 \mathrm{~mW}$ to $50 \mathrm{~mW}$.

Another two constants needed in this calculation are blood density (which is $1060 \mathrm{~kg} / \mathrm{m}^{3}$ [23]) and turbine efficiency (which can reach 90\%). These values, along with the relevant equations; were used in order to build a graph which demonstrates the relations between turbine diameter and electric output power. This graph is presented as Figure 2. One may see that; for example, approximately $0.9 \mathrm{~mm}$ diameter is required in order to illuminate at a power of $2 \mathrm{~mW}$. This is quite a small diameter but it is still relatively large for a turbine designed to be placed inside a blood vessel. In addition, the calculations we have made were based on blood velocity measured in the ascending aorta. As previously explained, type of blood vessel (artery or vein) as well as vessels' width affect blood velocity in such a way that the smaller the vessel is, the larger the required diameter is.

However, this does not really constitute a problem since the device we sought to develop is not designed to operate in a consecutive manner, and therefore, the electric power can be collected and used only when enough power is accumulated. This kind of adjustment will allow us to miniaturize the devices in such a manner that indeed will be suitable for blood vessel implantation (e.g., to have diameter of about $0.1 \mathrm{~mm})$.

In Figure 3(a), a graphic scheme of the intravascular implantable light therapeutic prototype is presented. As can be seen, a small propeller is positioned at one end of the device. The propeller is attached to a DC motor via an axis which crosses an isolating material consisting of a felt fabric socked in grease lubricant. In this prototype, the DC motor is used as a voltage generator for a LED source, located at the opposite end. All components (except the propeller) are placed inside an isolating polycarbonate cylinder to prevent water from entering. An image of the prototype is presented in Figure 3(b). Upon blood flow, the propeller turns around allowing the motor to generate electric power and activate the LED source.

After building the device, we sought to determine its electrical power. In order to do that, the device was placed 
inside a Perspex tube $(1.8 \mathrm{~cm}$ diameter $)$ which was connected to a water pump $(7000 \mathrm{~L} / \mathrm{h})$.

It is important to explain why we have chosen such a strong pump. As blood velocity in arteries is approximately $0.5 \mathrm{~m} / \mathrm{sec}$ and turbine diameter is two times smaller than the ascending aorta's diameter, a simple calculation will lead to the fact that in order to simulate blood velocity in ascending aorta inside the Perspex tube, a power of approximately $1000 \mathrm{~L} / \mathrm{h}$ is needed $\left(\pi r^{2} /(100 \times 5 \times 3600 \times 2)=916\right.$ with $r$ being the radius of the pipe). As our system suffers from a very high friction of the propeller's axis, we decided to take a bit stronger pump.

A $17.3 \mathrm{ohm}$ resistor was placed instead of the LED light source and the pump was activated. As can be seen in Figure 3(c), a voltage of $0.3 \mathrm{~V}$ was measured, indicating an electric power of $5 \mathrm{~mW}$.

\section{Conclusions}

In summary, in this paper we have presented preliminary results of the process of developing a novel self-powered light therapeutic device which is designed to be implanted inside a blood vessel and to implement blood irradiation therapy. This device will be self-powered by a miniaturized turbinebased generator which uses hydraulic energy in the form of blood flow as its power source.

First, we have found a function that describes blood velocity. Then, we have used this function along with relative equations to build a function which will be used in order to calculate the electric power that can be produced by blood flow during a single heart beat. In the next step, we have used these functions, along with real relevant parameters, in order to investigate feasibility and demonstrated the relations between turbine diameter and its produced electric power.

These calculations led to the conclusion that assuming the electric power can be collected and used only when enough power is accumulated, the device will indeed be suitable for blood vessel implantation.

Finally, we have presented a prototype of the device and conducted an experiment to measure its electrical power which was $5 \mathrm{~mW}$.

The research presented in this paper may become the first step towards a new type of biomedical self-operational micromechanical devices deployed for biomedical applications.

\section{References}

[1] J. C. Palmaz, "Balloon-expandable intravascular stent," American Journal of Roentgenology, vol. 150, no. 6, pp. 1263-1269, 1988.

[2] P. W. Serruys, P. De Jaegere, F. Kiemeneij et al., "A comparison of balloon-expandable-stent implantation with balloon angioplasty in patients with coronary artery disease," The New England Journal of Medicine, vol. 331, no. 8, pp. 489-495, 1994.

[3] J. C. Palmaz, "Intravascular stents in the last and the next 10 years," Journal of Endovascular Therapy, vol. 11, no. 2, pp. II200-II206, 2004.

[4] J. Greenhalgh, J. Hockenhull, N. Rao, Y. Dundar, R. C. Dickson, and A. Bagust, "Drug-eluting stents versus bare metal stents for angina or acute coronary syndromes," Cochrane Database of Systematic Reviews (Online), vol. 5, article CD004587, 2010.

[5] M. Bosiers, K. Deloose, J. Verbist, and P. Peeters, "Will absorbable metal stent technology change our practice?" Journal of Cardiovascular Surgery, vol. 47, no. 4, pp. 393-397, 2006.

[6] T. Ohki, D. Stern, M. Allen, and J. Yadav, "Wireless pressure sensing of aneurysms," Endovascular Today, pp. 47-52, 2004.

[7] T. Ohki, K. Ouriel, P. G. Silveira et al., "Initial results of wireless pressure sensing for endovascular aneurysm repair: the APEX Trial-Acute Pressure Measurement to Confirm Aneurysm Sac EXclusion," Journal of Vascular Surgery, vol. 45, no. 2, pp. 236242, 2007.

[8] E. B. Gelfgat, R. F. Abdullaev, Z. M. Babaev, and S. Musabekov Sh. S., "Helium-neon laser used in drug-resistant cardiac arrhythmias," Kardiologiya, vol. 32, no. 2, pp. 66-68, 1992.

[9] T. Moshkovska and J. Mayberry, "It is time to test low level laser therapy in Great Britain," Postgraduate Medical Journal, vol. 81, no. 957, pp. 436-441, 2005.

[10] G. E. Brill, S. N. Grigoriev, T. P. Romanova, and S. G. Petrisheva, "Effect of laser irradiation on neutrophils metabolism in stress," in Low-Energy Lasers, vol. 2086 of Proceedings of SPIE, pp. 312-318, 1993.

[11] E. Meshalkin, Application of Direct Laser Irradiation in Experimental and Clinical Heart Surgery, Nauka, Novosibirsk, Russia, 1981.

[12] E. A. Stroev, V. A. Larionov, L. P. Grigor'eva, V. G. Makarova, and I. I. Dubinina, "The treatment of diabetic angiopathies by endovascular low-intensity laser irradiation," Problemy Endokrinologii, vol. 36, no. 6, pp. 23-25, 1990.

[13] A. Dube, H. Bansal, and P. K. Gupta, "Modulation of macrophage structure and function by low level He-Ne laser irradiation," Photochemical and Photobiological Sciences, vol. 2, no. 8, pp. 851-855, 2003.

[14] H. Kolárová, D. Ditrichová, and S. Smolan, "Effect of $\mathrm{He}-\mathrm{Ne}$ laser irradiation on phagocytic activity of leukocytes in vitro," Acta Universitatis Palackianae Olomucensis Facultatis Medicae, vol. 129, pp. 127-132, 1991.

[15] A. I. Neľmark and N. I. Muzalevskaia, "Low-intensity laser radiation in preoperative preparation of patients with benign prostatic hyperplasia," Urologiia, no. 1, pp. 11-15, 2000.

[16] I. Stadler, R. Evans, B. Kolb et al., "In vitro effects of low-level laser irradiation at $660 \mathrm{~nm}$ on peripheral blood lymphocytes," Lasers In Surgery and Medicine, vol. 27, pp. 255-261, 2000.

[17] V. I. Karandashov, E. B. Petukhov, V. S. Zrodnikov, and V. A. Zhomov, "Biological and clinical effects of violet and blue light," Biulleten' Eksperimental'Noi Biologii I Meditsiny, vol. 123, no. 4, pp. 452-454, 1997.

[18] I. S. Diasamidze, "Impact of photo hemotherapy on blood lipid composition in coronary heart disease," Klinicheskaia Meditsina, vol. 82, no. 7, pp. 34-36, 2004.

[19] M. H. Weber, T. Fussgänger-May, and T. Wolf, "The intravenous laser blood irradiation-introduction of a new therapy," Deutsche Zeitschrift für Akupunktur, vol. 50, pp. 12-23, 2007.

[20] M. Beraia, "Arterial pulse impact on blood flow," Health, vol. 2, no. 6, pp. 532-540, 2010.

[21] L. J. Clancy, Aerodynamics, section 3.5, Pitman Publishing Limited, London, UK.

[22] N. Narendran, Y. Gu, J. P. Freyssinier-Nova, and Y. Zhu, "Extracting phosphor-scattered photons to improve white LED efficiency," Physica Status Solidi A, vol. 202, no. 6, pp. R60-R62, 2005.

[23] J. Cutnell and J. Kenneth, Physics, John Wiley \& Sons, New York, NY, USA, 4th edition, 1998. 

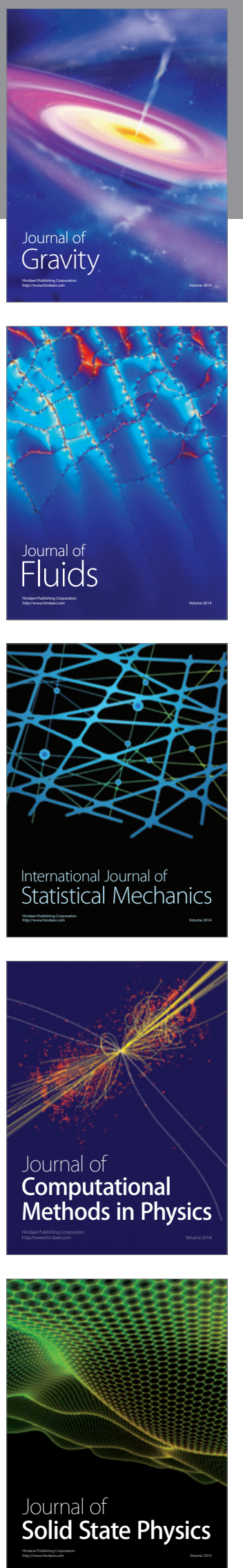

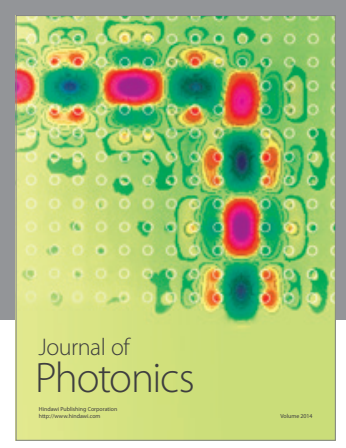

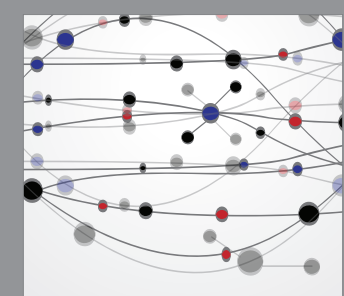

The Scientific World Journal
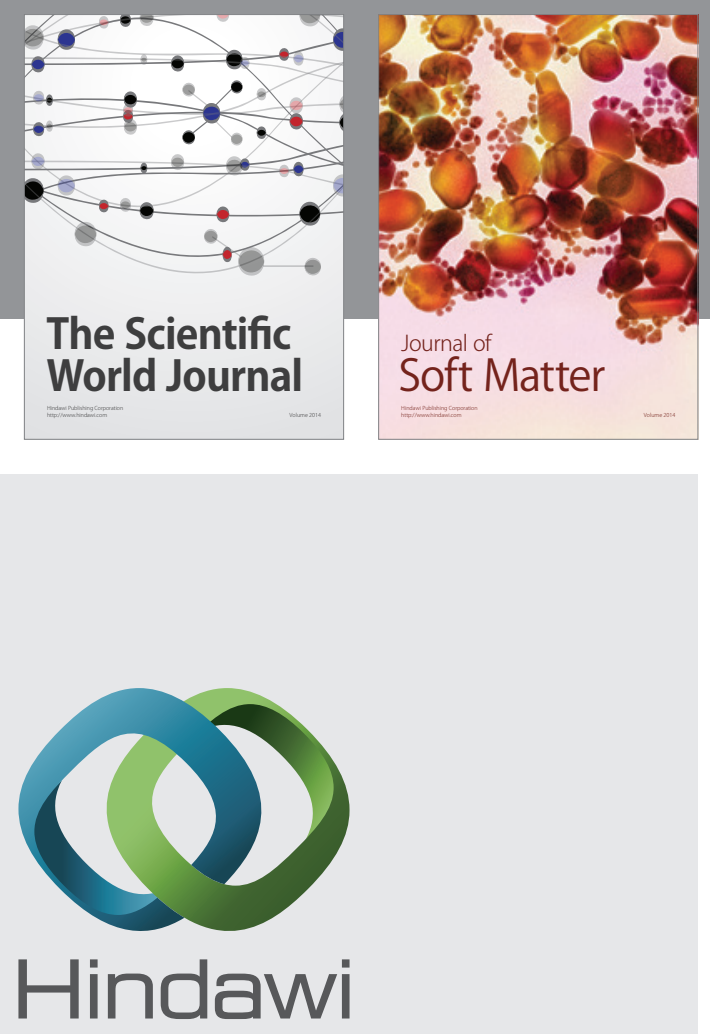

Submit your manuscripts at

http://www.hindawi.com
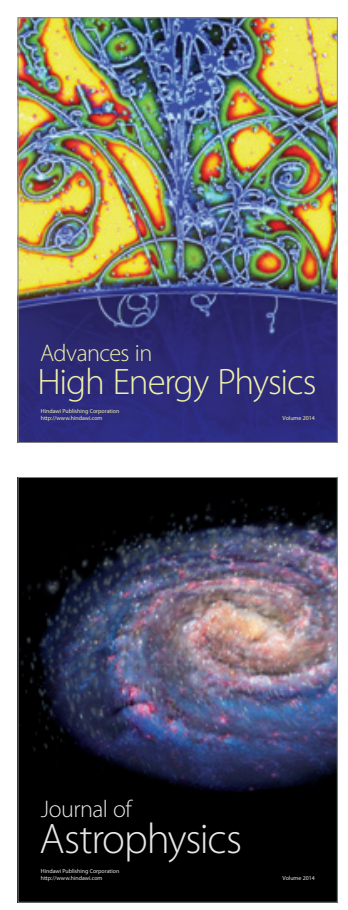
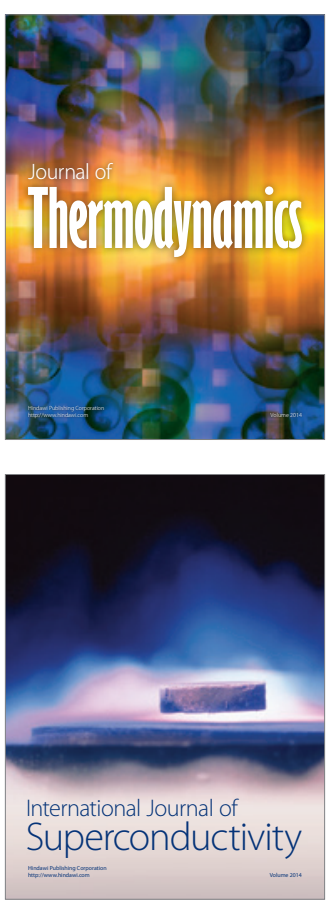
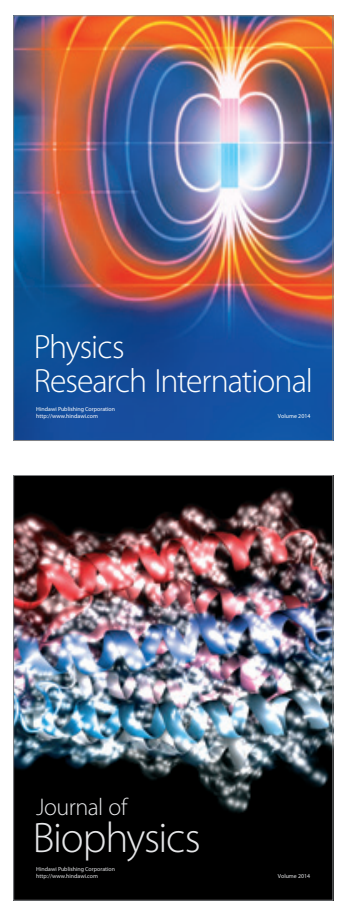
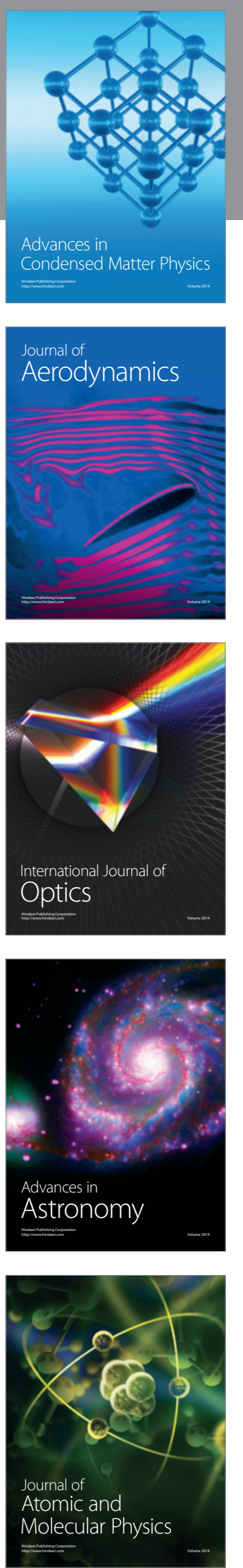